\title{
Crystal structure of 4-aza-2-(hydroxyimino)-3-methyl-5-(2-pyridyl)pent- 3-ene, $\mathrm{C}_{10} \mathrm{H}_{13} \mathrm{~N}_{3} \mathrm{O}$
}

\author{
J. Iulek'. J. Zukerman-Schpector ${ }^{* . I I I I I}$, C. C. Stadler', A. Tozetto' and M. Vega ${ }^{\text {II.III }}$ \\ I Universidade Estadual de Ponta Grossa - PR, Depto. Química. Av. Carlos Cavalcanti 4748. 84030-000 - Ponta Grossa, PR. Brazil \\ 1 Universidade Federal de São Cárlos. Depto. Química. Laboratório de Cristalografia, Estereodinâmica e Modelagem Molecular. \\ Caixa Postal 676.13565-905 - São Carlos, SP, Brazil \\ III Universidade de São Paulo. Instituto de Química. SP. Brazil
}

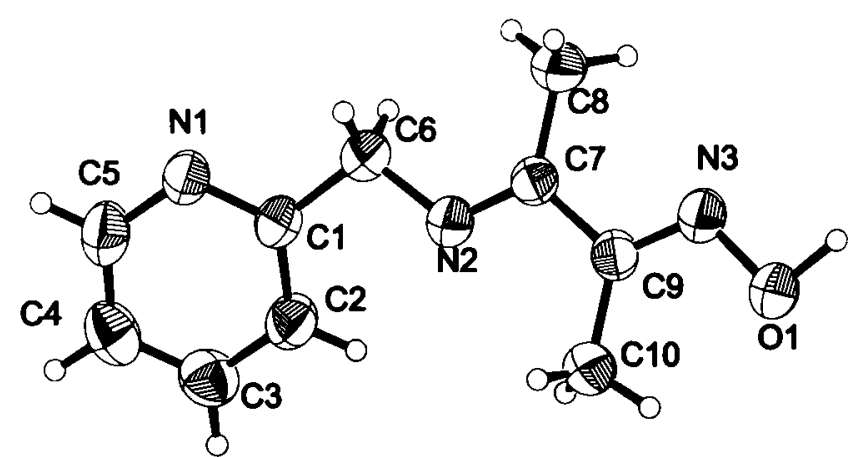

Abstract

$\mathrm{C}_{10} \mathrm{H}_{13} \mathrm{~N}_{3} \mathrm{O}$, monoclinic, $P 12_{1} / c 1$ (No. 14), $a=7.1477(9) \AA$, $b=18.768(2) \AA, c=7.651(1) \AA, \beta=91.36(1)^{\circ}, V=1026.1 \AA^{3}$, $Z=4, R_{\mathrm{gt}}(F)=0.045, w R_{\mathrm{all}}\left(F^{2}\right)=0.157, T=291 \mathrm{~K}$.

\section{Source of material}

$5 \mathrm{~g}(49.5 \mathrm{mmol})$ of biacethyl monoxime were dissolved in $20 \mathrm{~mL}$ of diisopropylic ether with $4.7 \mathrm{~mL}$ ( $5 \mathrm{~g}, 46.2 \mathrm{mmol}$ ) of 2-(2-aminomethyl)pyridine. The mixture was refluxed by one hour and cooled, then filtered and the resulting product washed with ethyl alcohol. Colourless needle crystals were obtained after recrystallization from ethanol at $277 \mathrm{~K}$. Crystals were separated by filtration, washed with ehtyl ether and dried under vacuum (mp $416 \mathrm{~K}$ ).

\section{Discussion}

The C6-C10,N2,N3.O1 moiety is almost planar (rms deviation of the best least square plane through them being $0.040 \AA$ ) making an angle of $28.39(6)^{\circ}$ with the pyridine ring. It is interesting to note that the molecule exhibits two intramolecular short contacts: C10-H10A $\cdots \mathrm{Ol}\left(d(\mathrm{H} \cdots \mathrm{O})=2.23 \AA ; \angle \mathrm{C}-\mathrm{H} \cdots \mathrm{O}=107^{\circ}\right)$ and $\mathrm{C} 2-\mathrm{H} 2 \cdots \mathrm{N} 2\left(d(\mathrm{H} \cdots \mathrm{N})=1.51 \AA, \angle \mathrm{C}-\mathrm{H} \cdots \mathrm{N}=99^{\circ}\right)$ which are responsible for the particular arrangement of the molecule. In order to study the influence of these short contacts in the molecular conformation a series of Potential Energy Surfaces (PES) calculations were performed (MOPAC7.01 [1,2] and GAMESS98 [3]). The geometry optimization calculations, using AMl and 6-31G*, showed that there is a change in the conformation, the $\mathrm{N} 2-\mathrm{C} 6-\mathrm{C} 1-\mathrm{N} 1$ torsion angle changes from $156.1(2)^{\circ}$ to $180^{\circ}$. This involves the $\mathrm{C} 2-\mathrm{H} 2 \cdots \mathrm{N} 2$ interaction, in fact the $\mathrm{H} 2 \cdots \mathrm{N} 2$ distance shortens from $2.51 \AA$ to $2.48 \AA$. As the molecules are joined through a classical hydrogen bond involving the pyridyl $\mathrm{N}$ atom and the hydroxyl group: $d\left(\mathrm{H} 101 \cdots \mathrm{N} l^{i}\right)=1.66 \AA$, $\angle \mathrm{O} 1-\mathrm{H} 1 \mathrm{O} 1 \cdots \mathrm{N} 1^{i}=177^{\circ}(i=1-x, 0.5+y, 0.5-z)$. We can postulate that, in this case, the molecular conformation is driven more by the intermolecular interaction. Whether all the mentioned $\mathrm{C}-\mathrm{H} \cdots \mathrm{X}$ interactions are true hydrogen bonds is difficult to assert because, as pointed out by Cotton et al. [4], 'the field is getting muddier and muddier as the definition of a hydrogen bond is relaxed'. In any case, we have only take into account those with $\mathrm{H} \cdots \mathrm{X}$ distance less than the sum of the van der Waal's radii [5] plus $10 \%$ and with an $\mathrm{C}-\mathrm{H} \cdots \mathrm{O}$ angle greater than, or very close to $100^{\circ}$.

Table 1. Data collection and handling.

\begin{tabular}{ll}
\hline Crystal: & $\begin{array}{l}\text { colourless, irregular, } \\
\text { size } 0.10 \times 0.20 \times 0.25 \mathrm{~mm}\end{array}$ \\
Wavelength: & Mo $K_{\alpha}$ radiation $(0.71073 \AA)$ \\
$\mu:$ & $0.84 \mathrm{~cm}^{-1}$ \\
Diffractometer, scan mode: & CAD-4 Mach $3, \omega / 2 \theta$ \\
$2 \theta_{\text {max }}:$ & $51.8^{\circ}$ \\
$N(h k l)_{\text {measured, }} N(h k l)_{\text {unique }}:$ & 2129,1980 \\
Criterion for $I_{\text {obs, }} N(h k l)_{\text {gl }}:$ & $I_{\text {obs }}>2 \sigma\left(I_{\text {obs }}\right), 1070$ \\
$N(p a r a m)_{\text {refined: }}$ & 129 \\
Programs: & SIR92 [6], MolEn [7], SHELXL-97 [8] \\
& PARST95[9], ZORTEP [10], WinGX [11] \\
& PLATON [12]
\end{tabular}

Table 2. Atomic coordinates and displacement parameters (in $\AA^{2}$ ).

\begin{tabular}{llllll}
\hline Atom & Site & $x$ & $y$ & $z$ & $U_{\text {iso }}$ \\
\hline H(1O1) & $4 e$ & 0.4774 & 0.6749 & 0.3921 & 0.075 \\
H(6A) & $4 e$ & 0.4794 & 0.3494 & 0.0214 & 0.060 \\
H(6B) & $4 e$ & 0.5113 & 0.3198 & 0.2119 & 0.060 \\
H(5) & $4 e$ & 0.8199 & 0.1355 & 0.0093 & 0.072 \\
H(10A) & $4 e$ & 0.8604 & 0.5825 & 0.3170 & 0.090 \\
H(10B) & $4 e$ & 0.8760 & 0.5232 & 0.1740 & 0.090 \\
H(10C) & $4 e$ & 0.8725 & 0.5022 & 0.3722 & 0.090 \\
H(8A) & $4 e$ & 0.2805 & 0.4164 & 0.1434 & 0.089 \\
H(8B) & $4 e$ & 0.2652 & 0.4987 & 0.1749 & 0.089 \\
H(8C) & $4 e$ & 0.2797 & 0.4465 & 0.3345 & 0.089 \\
H(3) & $4 e$ & 1.1404 & 0.2998 & -0.1124 & 0.073 \\
H(2) & $4 e$ & 0.9076 & 0.3750 & -0.0154 & 0.067 \\
H(4) & $4 e$ & 1.0971 & 0.1774 & -0.0928 & 0.076 \\
& & & & & \\
\hline
\end{tabular}

* Correspondence author (e-mail: julio@power.ufscar.br) 
Table 3. Atomic coordinates and displacement parameters (in $\AA^{2}$ ).

\begin{tabular}{|c|c|c|c|c|c|c|c|c|c|c|}
\hline Atom & Site & $x$ & $y$ & $=$ & $U_{11}$ & $U_{22}$ & $U_{33}$ & $U_{12}$ & $U_{13}$ & $U_{23}$ \\
\hline$O(1)$ & $4 e$ & $0.5960(2)$ & $0.64319(8)$ & $0.3570(2)$ & $0.056(1)$ & $0.0436(9)$ & $0.089(1)$ & $-0.0010(7)$ & $0.0041(9)$ & $-0.0137(9)$ \\
\hline$N(3)$ & $4 e$ & $0.5032(3)$ & $0.58012(9)$ & $0.3090(2)$ & $0.052(1)$ & $0.041(1)$ & $0.057(1)$ & $-0.0002(8)$ & $0.0047(9)$ & $-0.0023(8)$ \\
\hline$N(2)$ & $4 e$ & $0.6418(2)$ & $0.41251(9)$ & $0.1715(2)$ & $0.047(1)$ & $0.040(1)$ & $0.056(1)$ & $-0.0004(8)$ & $0.0045(8)$ & $-0.0038(9)$ \\
\hline$N(1)$ & $4 e$ & $0.7019(2)$ & $0.22682(9)$ & $0.0587(2)$ & $0.054(1)$ & $0.038(1)$ & $0.052(1)$ & $-0.0004(8)$ & $-0.0023(8)$ & $0.0001(8)$ \\
\hline$C(1)$ & $4 e$ & $0.7292(3)$ & $0.2974(1)$ & $0.0528(3)$ & $0.050(1)$ & $0.039(1)$ & $0.038(1)$ & $-0.0025(9)$ & $0.0001(9)$ & $-0.0001(9)$ \\
\hline$C(7)$ & $4 e$ & $0.5290(3)$ & $0.4611(1)$ & $0.2173(3)$ & $0.042(1)$ & $0.040(1)$ & $0.043(1)$ & $-0.0002(9)$ & $0.0065(9)$ & $0.0048(9)$ \\
\hline$C(6)$ & $4 e$ & $0.5717(3)$ & $0.3432(1)$ & $0.1152(3)$ & $0.048(1)$ & $0.043(1)$ & $0.060(1)$ & $-0.004(1)$ & $0.005(1)$ & $0.001(1)$ \\
\hline$C(9)$ & $4 e$ & $0.6163(3)$ & $0.5295(1)$ & $0.2741(3)$ & $0.045(1)$ & $0.04 !(1)$ & $0.046(1)$ & $0.0025(9)$ & $0.0053(9)$ & $0.0021(9)$ \\
\hline$C(5)$ & $4 e$ & $0.8385(4)$ & $0.1845(1)$ & $0.0044(3)$ & $0.073(2)$ & $0.041(1)$ & $0.067(2)$ & $0.006(1)$ & $-0.002(1)$ & $-0.007(1)$ \\
\hline$C(10)$ & $4 e$ & $0.8250(3)$ & $0.5348(1)$ & $0.2853(3)$ & $0.048(1)$ & $0.052(1)$ & $0.080(2)$ & $0.001(1)$ & $0.006(1)$ & $-0.011(1)$ \\
\hline$C(8)$ & $4 e$ & $0.3198(3)$ & $0.4552(1)$ & $0.2175(3)$ & $0.046(1)$ & $0.056(1)$ & $0.077(2)$ & $-0.004(1)$ & $0.011(1)$ & $-0.008(1)$ \\
\hline$C(3)$ & $4 e$ & $1.0307(3)$ & $0.2812(1)$ & $-0.0680(3)$ & $0.055(2)$ & $0.068(2)$ & $0.058(2)$ & $0.002(1)$ & $0.014(1)$ & $-0.003(1)$ \\
\hline $\mathrm{C}(2)$ & $4 e$ & $0.8917(3)$ & $0.3258(1)$ & $-0.0109(3)$ & $0.058(1)$ & $0.046(1)$ & $0.063(2)$ & $-0.004(1)$ & $0.012(1)$ & $0.002(1)$ \\
\hline$C(4)$ & $4 e$ & $1.0046(4)$ & $0.2089(1)$ & $-0.0581(3)$ & $0.064(2)$ & $0.065(2)$ & $0.061(2)$ & $0.017(1)$ & $0.004(1)$ & $-0.011(1)$ \\
\hline
\end{tabular}

Acknowledgments. This work has received parial suppon from FAPESP, CNPq, CAPES and FINEP. The $X$-ray facility at the Instituto de Química-USP was established with the assistance of FAPESP(94/2061-4).

\section{References}

1. Csern, I.: MOPAC7.01 for linux (public domain version). Institute of Nucl. Research of Hung. Acad. Sci. H-4001 Debrecen POB 51, Hungary.

2. Stewart, J. J. P.: MOPAC: A Semiempirical Molecular Orbital Program. J. Comput. Aid. Mol. Des. 4 (1990) 1-105.

3. Schmidt, M. W.; Baldridge, K. K.; Boatz, J. A.; Elbert, S. T., Gordon, M. S.; Jensen, J. H.; Koseki, S.; Matsunaga, N.; Nguyen, K. A.; Su, S. J.; Windus, T. S.; Dupuis, M.; Montgomery, J. A : GAMESS98: J. Comput. Chem. 14 (1993) 1347-1363.

4. Cotton, F. A.; Daniels, L. M.; Jordan, G. T.; Murillo, C. A.: The crystal packing of bis(2,2'-dipyridylamido)cobalt(II), $\mathrm{Co}(\mathrm{dpa})_{2}$, is stabilized by $\mathrm{C}-\mathrm{H} \cdots \mathrm{N}$ bonds: are there any real precedents? J. Chem. Soc. Chem. Commun. (1997) 1673-1674.

5. Paulig, L.: The Nature of the Chemical Bond. 3rd. Ed., Comell University Press, Ithaca, NY 1960.

6. Altomare, A.; Cascarano, G.; Giacovazzo, C.; Guagliardi, A.; Completion and refinement of crystal structures with SIR92. J. Appl. Crystallogr. 26 (1993) 343-350.

7. Fair, C. K.: MolEn. An Interactive Intelligent System for Crystal Structure Analysis. Enraf-Nonius, Delft, The Netherlands 1990.

8. Sheldrick, G. M.: SHELXL-97. Program for the Refinement of Crystal Structures. University of Göttingen, Germany 1997.

9. Nardelli, M.: PARST95 - an update to PARST: a system of Fortran routines for calculating molecular structure parameters from the result of crystal structure analyses. J. Appl. Crystallogr. 28 (1995) 659.

10. Zsolnai, L.: ZORTEP. An Interactive Molecular Graphics Program. University of Heidelberg, Germany 1995.

1 1. Farrugia, L. J.: WinGX suite for small-molecule single-crystal crystallography. J. Appl. Crystallogr. 32 (1999) 837-838.

12. Spek, A.L.: PLATON, A Multipurpose Crystallographic Tool. Utrecht University, Utrecht. The Netherlands 1998. 